\title{
Investigations on the characterization of ion implanted hexagonal boron nitride
}

\author{
E. Aradi, S.R. Naidoo, R.M. Erasmus, B. Julies and T.E. Derry
}

\begin{abstract}
The effect of ion implantation on hexagonal boron nitride (h-BN) is studied herein. We use boron as an ion of choice to introduce radiation damage into $\mathrm{h}-\mathrm{BN}$, at fluences ranging from $1 \times 10^{14-1} \times 10^{16}$ ions $/ \mathrm{cm}^{2}$ and implantation energy ranges from 40 to $160 \mathrm{keV}$. The thermal dependence is also investigated by varying the annealing temperature from room temperature to $400{ }^{\circ} \mathrm{C}$ after implantation. Raman spectroscopy showed Raman active defects one of which is possibly related to the formation of cubic boron nitride nanocrystals (nc-BN) within the implanted range. The relationship of these defect induced Raman active peaks was investigated by varying the implantation parameters. The preliminary Transmision Electron Microscopy (TEM) results also are reported briefly.
\end{abstract}

\section{Introduction}

Boron nitride (BN) is an interesting group $I I I-V$ binary com- pound; formed from atoms that straddle carbon in the periodic table, it occurs in modifications that are isostructural to those formed by carbon. The hexagonal BN (similar to graphite) and cubic BN (similar to diamond) phases are the stable phases and therefore have numerous applications. Synthesis of $c$-BN has been found to be of interest because of its excellent physicochemical properties, some of which are superior to those of diamond [1,2]. Hexagonal BN also exhibits extreme properties especially where thermal stability and chemical inertness are concerned $[3,4]$. The production of a thin film $c$-BN layer on $h$-BN surface would have advantages in combining the properties of both materials. Hexagonal $\mathrm{BN}$ has been used as a starting material in the phase change of various phases of $\mathrm{BN}[5]$.

Both theory and experiments have shown that defects induced into $h$-BN can enhance a phase change to the cubic form [6,7]. This work focuses on the role of point defects induced by using ion implantation on $h$-BN with an aim of transforming it to the cubic $\mathrm{BN}$ form. We aim at establishing the relationship and trend of various irradiation parameters with respect to the defects that are created and how they affect this transformation. The parameters that are investigated include the ion fluence, the ion energy and the role of annealing on the implanted substrate. We aim at optimizing these conditions to attain the most favorable 
conditions for the optically Raman active bands which may be ascribed to a trigonal symmetry.

\section{Experiments}

Single crystal $h$-BN samples, supplied by Goodfellow Ceramic Company, were used as substrates throughout this work. The samples had dimensions of $5 \times 5 \times 0.2$ $\mathrm{mm}$, with a $0.5 \mathrm{~lm}$ surface roughness. Implantation experiments were carried out using the 200-20A2F ion implanter, located at iThemba LABS (Gauteng), South Africa, with an implantation procedure similar to those in [8]. In this study we consider the effect of varying the boron energy and dose. Boron ion was chosen since it is one of the atomic species of $\mathrm{BN}$ and hence does not alter the chemical composition of the material after implantation.

Raman spectroscopy and some preliminary Transmission Electron Microscopy (TEM) were used for analysis. Raman measurements were carried out with the green laser $(514 \mathrm{~nm})$ line. The samples for TEM analysis were prepared using Focused Ion Beam (FIB) sectioning, at the Council of Scientific and Industrial Research (CSIR), South Africa, to obtain electron transparent lamellae, with platinum used as a coating.

\section{Results and discussions}

\subsection{Implantation dose effect}

Fig. 1 shows the Raman spectra for $h$-BN samples before and after implantation with boron ions at fluences of $1 \times 10^{14}, 5 \times 10^{14}$ and $1 \times 10^{15} \mathrm{ions} / \mathrm{cm}^{2}$ at an energy of $150 \mathrm{keV}$, and the spectrum for $c$-BN powder.

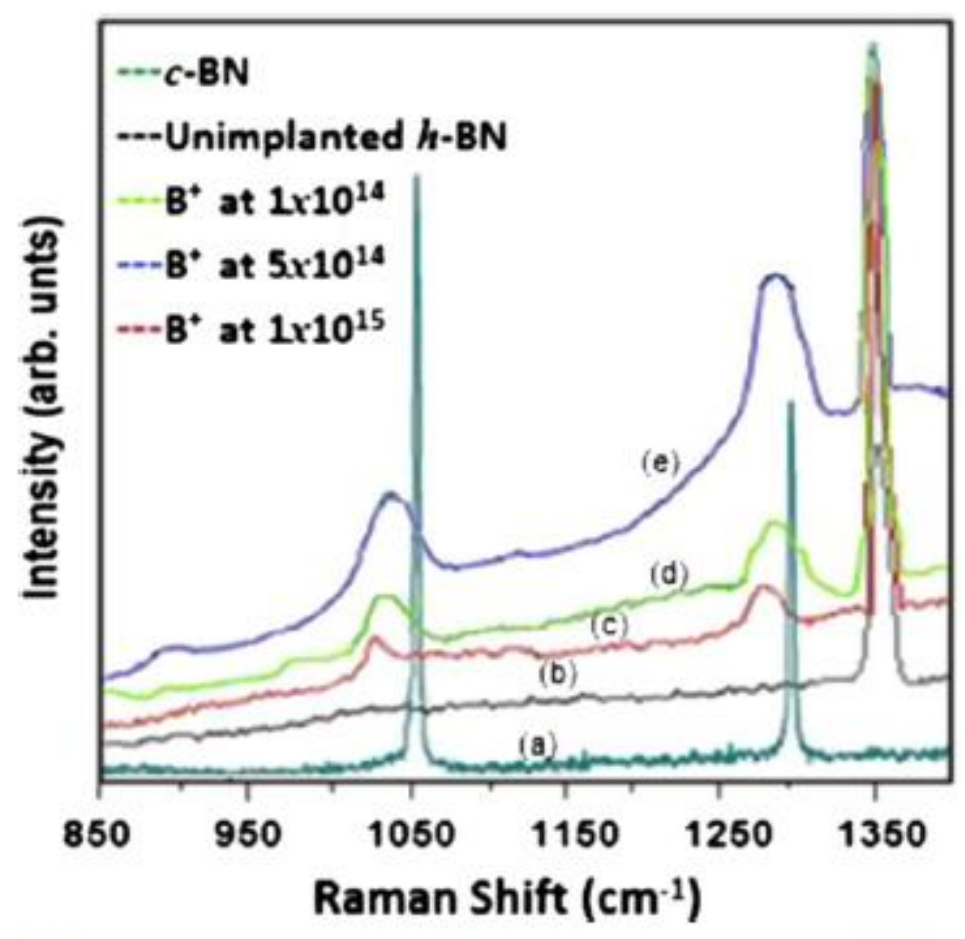

Fig. 1. $\mu$-Raman spectra for (a) c-BN powder, (b) single crystal $h$-BN before implantation (c), (d), and (e) $h$-BN after boron ion implantation with fluences of $1 \times 10^{14}, 1 \times 10^{15}$ and $5 \times 10^{14}$ ions $/ \mathrm{cm}^{2}$, respectively. 
The unimplanted spectrum shows a peak centered at wavenumber $1366 \mathrm{~cm}^{-}$ 1, with a FWHM of $8.1 \mathrm{~cm}^{-1}$, which normally represents the $E_{2 g}$ high frequency mode for pure $h-\mathrm{BN}$. The $c$-BN spectrum shows two peaks at 1054 and $1305 \mathrm{~cm}^{-1}$. These peaks represent the Transverse Optical (TO) and the Longitudinal optical (LO) Raman modes for $c-\mathrm{BN}$, respectively. The presence of these phonon modes is very much dependent on the crystal size for c-BN. The TO mode is normally absent in nanosized $c-B N$ crystals due to the selection rules with respect to crystal size in line with phonon confinement model [9].

After implantation the principal $h$-BN peak is still predominant in the spectrum but its intensity decreases and slightly broadens with increasing fluence. Its position does not change after implantation. We do note that a significant portion of the Raman scattering does emerge from the unimplanted substrate beyond the end of range of the implanted ions and damage profile. The implanted spectra also show emergence of peaks that are absent in the unimplanted spectrum at wavenumbers 1290, 1302 and $1296 \mathrm{~cm}^{-1}$ for the three fluences, respectively. The samples receiving $5 \times 10^{14}$ ions $/ \mathrm{cm}^{2}$ had the highest peak, and narrowest linewidth as compared to the other fluences indicating that the size and symmetry of the peaks are dependent on the ion fluence. Additional peaks are also observed in the implanted spectra, centered at 1022,1033 and $1025 \mathrm{~cm}^{-1}$, for the respective fluences. These peaks are absent in $h$-BN samples implanted with other ions such as He and Li reported in [8].

The new peaks around $1300 \mathrm{~cm}^{-1}$ in Fig. 1 for implanted samples are synonymous to the LO peak for $c-\mathrm{BN}$ indicating a possible phase change to $c$ - $\mathrm{BN}$. It has been observed that for nanocrystalline $c-\mathrm{BN}$, there occurs an unstructured peak, with characteristic asymmetric broadening and a frequency downshift close to the LO mode for $c$-BN [9]. These characteristics are similar to the ones observed in the implanted spectra; therefore, we attribute these peaks to $c-\mathrm{BN}$ nanosized crystals. Theory has shown that the presence of an interstitial between the basal layers of $h$-BN leads to buckling of the weak Van der Waals bonds which leads to a bond reconstruction to form covalent B-N bond with $s p 3$ hybridization present in $c$-BN [6]. We therefore attribute this phase transformation to originate from point defects introduced into the $h$-BN sample after implantation.

The additional phonon bands at 1022, 1033 and $1025 \mathrm{~cm}^{-1}$ have been observed in boron rich solids [10] with no boron peaks observed close to the $c$ $\mathrm{BN}$ phonon bands at $1305 \mathrm{~cm}^{-1}$. We do not attribute these peaks to the TO phonon mode; this is because, the TO mode is observed on bulk c-BN samples and it is highly unlikely that implantation is creating microcrystalline $c-\mathrm{BN}$ particles. It is more probable therefore that these Raman bands are related to B inclusions in the boron implanted $h$-BN crystal samples. 


\subsection{Energy dependence}

The effect of varying the implantation energy was investigated. The intensity of the principal $h$-BN peak decreases with increasing energy, while its FWHM and position remain constant. Fig. 2(a) shows the $c$-BN LO Raman spectra for samples that were implanted with boron at fluence of $5 \times 10^{14}$ ions $/ \mathrm{cm}^{2}$, in the energy range of 40-160 keV. Fig. 2(b) shows the relationship of the integrated intensity of the LO $c$-BN peak and the linewidth with the implantation energy. The integrated intensity and the linewidth are observed to increase with increasing energy. There is also a slight downshift in the frequency with the highest shift observed at the highest implant energy.

The peak broadening may be stress related. With increasing energy, the implanted layer is deeper below the crystal and as a result the induced stress will be dependent on how far the implanted layer is below the surface. This is observed in [11]. An alternative plausible explanation could be that as a result of the implanted layer being deeper at higher energies, the width of the electronic stopping region is higher. The defects that result in the $n c-B N$ formation could be created in the electronic stopping region. If this is the case then the observed broadening of the vibrational band is possibly from the smaller crystallites created in the electronic stopping region.

\subsection{Temperature dependence}

The temperature dependence of the linewidth of implanted $h$ - BN was studied and results are shown in Fig. 3. After implantation with boron at $120 \mathrm{keV}$ and fluence of $5 \times 10^{14}$ ions $/ \mathrm{cm}^{2}$, the samples were annealed at temperatures ranging from 50 to $400{ }^{\circ} \mathrm{C}$ for $120 \mathrm{~s}$ for the different temperatures. This was compared to the samples that were implanted at room temperature of $23{ }^{\circ} \mathrm{C}$.

From Fig. 3, it is indicated that the linewidth of the c-BN LO peak decreased with increasing annealing temperature, showing a temperature-induced Raman peak narrowing with increasing annealing temperature. The elevated temperature reduced the amount of damage or stress as monitored by the linewidth. More pertinently, the resultant defect ascribed to the $c$-BN is stable at up to the maximum annealing temperature. Analysis for samples annealed at higher temperatures above $400{ }^{\circ} \mathrm{C}$ was hindered due to a white residue forming on the annealing furnace within the Raman Spectroscope interfering with the measurements. The line- width and frequencies of the principal $h$-BN peak remained unaltered.

\section{Preliminary TEM results}

Fig. 4 shows the TEM preliminary results for an $h$-BN sample that was implanted with boron at $5 \times 10^{14}$ ions $/ \mathrm{cm}^{2}$ and the energy of $160 \mathrm{keV}$. The sample was prepared using the FIB technique. Fig. 4(a) represents the conventional TEM image for the whole sample. Region 1 is the substrate while region 2 shows a continuous layer created during implantation. The width of this 
layer corresponds to the range of implantation for $\mathrm{B}$ at $160 \mathrm{keV}$, calculated by SRIM simulations.
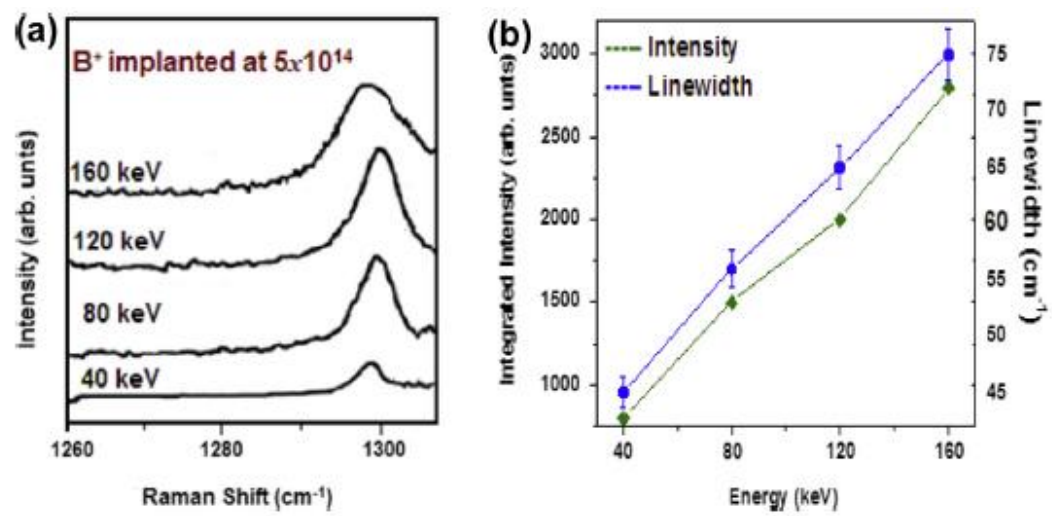

Fig. 2. (a) $\mu$-Raman spectra for $h$-BN samples implanted with boron at $5 \times 10^{14}$ ions $/ \mathrm{cm}^{2}$, at different energies. (b) Energy relationship with the peak's integrated intensity and the linewidth.

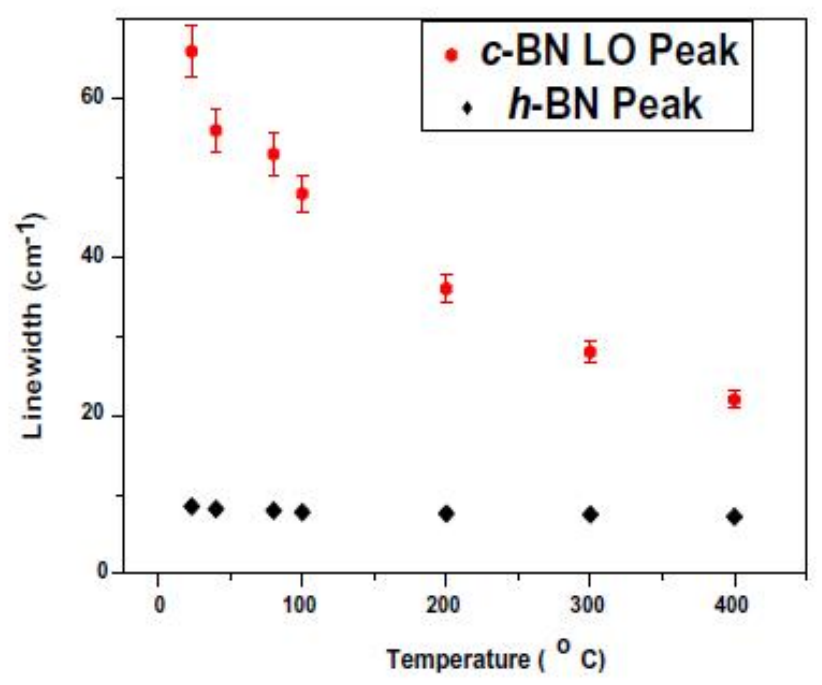

Fig. 3. Relationship between the annealing temperature and the linewidth of the LO $c$-BN peak and the principal $h$-BN peak for B implanted samples at $120 \mathrm{keV}$ and fluence of $5 \times 10^{14}$ ions $/ \mathrm{cm}^{2}$.

(a)

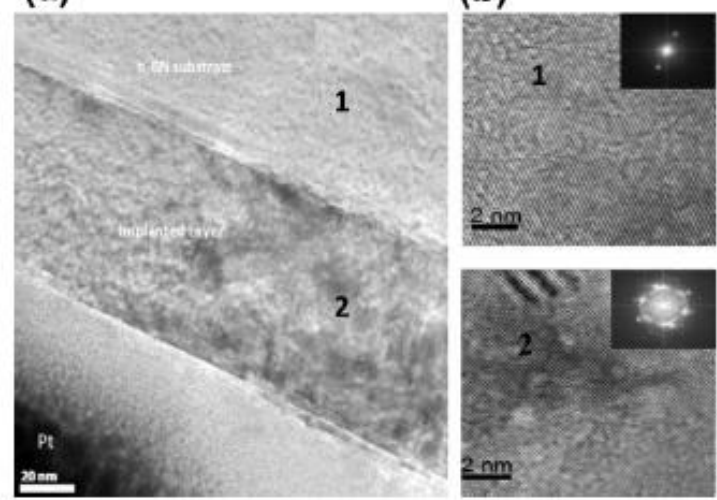

Fig. 4. (a) Cross-sectional TEM for samples implanted with boron at $160 \mathrm{keV}$ and fluences of $5 \times 10^{14}$ ions $/ \mathrm{cm}^{2}$. (b) HRTEM for unimplanted and implanted regions. The insets show the FFT images for these regions. 
Fig. 4(b) represents the HRTEM carried out in regions 1 and 2. The inset shows the Fourier transform images for these regions. The FFT images for region 1 show a crystalline diffraction pattern for $\mathrm{h}-\mathrm{BN}$, indicating that the substrate is entirely h-BN. The FFT for region 2 show a diffraction pattern of both amorphous and crystalline phases. The crystalline phase represents the image formed by c-BN with (111) reflection [12]. This work is still continuing.

\section{Conclusion}

An ion induced phase change of h-BN to c-BN nanocrystals is evident from the broad c-BN LO phonon peaks present for samples after implantation. The phase change was attributed to point defects introduced into the samples by ion implantation. The broadening and the frequency shift of the peaks from the known c-BN LO mode that occurs at $1305 \mathrm{~cm}^{-1}$ was attributed to the presence of the nanosized c-BN in line with the Phonon Confinement effect. The phase change was dependent on the fluence with an optimum at $5 \times 10^{14} \mathrm{ions} / \mathrm{cm}^{2}$. Annealing after implantation reduced the stress in the implanted layer as seen in the reduction in the line-width of the c-BN peak with increasing temperature. TEM preliminary results show the presence of nanocrystalline c$\mathrm{BN}$ phase in the implanted layer of h-BN complementing results from Raman spectroscopy. Further work will be directed towards using other methods such as GIXRD and FTIR to characterize the material, and to quantify the amount of the h-BN that has been transformed to c-BN.

\section{Acknowledgements}

The authors are grateful to the DST/NRF Centre of Excellence in Strong Materials and the University of the Witwatersrand for financial support, and iThemba LABS (Gauteng) for the implanter facility. 


\section{References}

[1] Q. Li, S.F. Wong, W.M. Lau, C.W. Ong, Diamond Relat. Mater. 16 (2007) 421.

[2] H. Uchida, M. Yamashita, S. Hanaki, A. Kurihara, Mater. Sci. Eng. A (2008) 695 .

[3] O. Lehtinen, T. Nikitin, A.V. Krasheninnikov, Phys. Status Solidi C 7 (2010) 1256.

[4] I.M. Joni, R. Balgis, T. Ogi, T. Iwaki, K. Okuyama, Colloids Surf. Physicochem. Eng. 388 (2011) 49.

[5] A.F. Jankowski, J.P. Hayes, D.M. Makowiecki, M.A. McKernan, Thin Solid Films 308 (1997) 94.

[6] T.E. Mosuang, T. Lowther, Phys. Rev. B 63 (2002) 014112.

[7] R. Machaka, R.M. Erasmus, T.E. Derry, Diamond Relat. Mater. 19 (2010) 11331.

[8] E. Aradi, R.M. Erasmus, T.E. Derry, Nucl. Instr. Meth. Phys. Res. B 27 (2) (2012) 57.

[9] T. Werninghaus, M. Friedrich, J. Hahn, M. Röder, F. Richter, D.R.T. Zahn, Appl. Phys. Lett. 70 (1997) 958.

[10] C.I. Beckel, M. Yousaf, M.Z. Fuka, F.Y. Rasa, N. Li, Phys. Rev. B 44 (1991) 2535 .

[11] M. Benzohra, F. Olivie, M.I. Benzohra, K. Ketata, M. Ketata, Nucl. Instr. Meth. Phys. Res. B 18 (7) (2002) 201.

[12] S. Kidner, C.A. Taylor II, R. Clarke, Appl. Phys. Lett. 64 (14) (1994) 1859. 